\title{
Políticas sociales en la coyuntura de pandemia por COVID-19: el rol del Trabajo Social en el contexto costarricense.
}

\section{Social policies in the COVID-19 pandemic situation: the role of Social Work in the Costa Rican context.}

\author{
Políticas sociais na situação da pandemia COVID-19: o \\ papel do Serviço Social no contexto costarriquenho.
}

\author{
Laura Rivera Alfaro* \\ Mariangel Sánchez Alvarado*
}

\begin{abstract}
Resumen: El texto tiene como objetivo evidenciar las demandas emergentes colocadas por la pandemia de la COVID-19 en la respuesta del Estado. Además, mencionar algunas estrategias utilizadas por las personas profesionales en trabajo social en la formación social costarricense, inmersas en una coyuntura de precarización de las condiciones de vida de la población desde la crisis estructural del capital y agravada en el contexto de la pandemia. Este texto se realizó a partir de una pesquisa bibliográfica y documental, utilizando fuentes primarias de información como noticias de periódicos, información del Colegio Profesional de Trabajo Social de Costa Rica y documentos públicos institucionales con datos sobre el tema. Con base en la información recabada y el análisis realizado, se evidencia una creciente pobreza y desigualdad vivida no solamente en Costa Rica, sino por toda la región de América Latina. Debido a su condición de países periféricos, vinculados orgánicamente a un sistema de producción mundial capitalista, se ha generado un detrimento en las condiciones de vida de la clase trabajadora, especialmente en las condiciones de pobreza, empleo, acceso a la salud y educación. Tales condiciones son agudizadas en el contexto actual de desmonte de los derechos sociales, donde
\end{abstract}

\footnotetext{
* Máster en Servicio Social, Universidad Federal de Pará. Licenciada en Trabajo Social, Universidad de Costa Rica, Licenciada en Derecho, Universidad Latina de costa Rica. Consultora independiente. lau.riveralfaro@gmail.com

** Doctoranda en Servicio Social, Universidad Federal de Alagoas. Maestría en Política Social, Universidad Federal de Mato Grosso. Licenciada en Trabajo Social, Universidad de Costa Rica. Coordinadora Unidad de Investigación, Colegio de Trabajo Social de Costa Rica. Docente en la Escuela de Trabajo Social, Universidad de Costa Rica. mariangelsa@gmail.com.
}

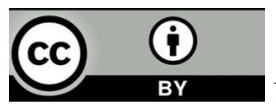


trabajo social como profesión articulada intrínsecamente a la reproducción del modo de producción por medio de la política social y como profesión que atiende directamente las manifestaciones de la cuestión social ha tenido que idear estrategias de atención.

Palabras claves: Práctica profesional. Políticas públicas. Costa Rica.

\begin{abstract}
The text aims to demonstrate the emerging demands placed by the COVID-19 pandemic in the response of the State, mentioning some strategies used by professionals in Social Work in the Costa Rican socialtraining, immersed in a situation of precariousness of the living conditions of the population since the structural crisis of capital and aggravated in the context of the pandemic. The article was made from the documentary research, using primary sources of information such as news, information from the professional association of Social Work of Costa Rica and public documents with data on the subject. Based on the information collected and the analysis carried out, it is evident how the growing poverty and inequality experienced not only in Costa Rica, but throughout the Latin American region due to their condition as peripheral countries, organically linked to a capitalist production system On a world scale, it has generated a detriment to the living conditions of the working class, especially poverty, employment, access to health and education. Such conditions are exacerbated in the current context where social work as a profession articulated inherently to mode of production through social policy and as a profession that directly addresses the manifestations of the social question, has had to devise strategies of care in a context of dismantling of social rights.
\end{abstract}

Keywords: Professional practice. Public politics. Costa Rica.

Resumo: O texto tem como objetivo evidenciar as demandas emergentes colocadas pea pandemia da COVID-19 na resposta do Estado, fazendo ênfase em algumas estratégias utilizadas pelos Trabalhadores Sociais (Assistentes Sociais) na formação social costarriquenha, imersas na conjuntura de precarização das condições de vida da população desde a crise estrutural do capital e agravada no contexto da pandemia. 0 artigo foi realizado a partir da pesquisa bibliográfica e documental, utilizando fontes primárias de informação como notícias de jornais, informação do Colégio profissional de Trabalhadores Sociais da Costa Rica e documentos públicos com dados sobre o tema. Baseando-se na informação coletada e a análise realizada, evidenciase como o aumento da pobreza e a desigualdade vivenciada não somente na Costa Rica, senão também na região da América Latina e Caraíbas pela sua condição de países periféricos, vinculados organicamente a um sistema de produção mundial capitalista, tem gerado um detrimento das condições de vida da classe trabalhadora, especialmente na pobreza, desemprego, acesso à saúde e educação. Tais condições são agudizadas no contexto atual, onde o Trabalho Social (Serviço Social), como profissão articulada intrinsecamente à reprodução do capitalismo mediante a política social e como profissão que 
intervém diretamente nas manifestações da questão social, tem tido que idear estratégias de atenção nesse contexto de pandemia.

Palavras chaves: Prática profissional. Políticas públicas. Costa Rica.

Recebido em 21/06/2021. Aceito em: 05/10/2021

\section{Introducción}

Este artículo tiene como propósito presentar un acercamiento a la coyuntura actual de la formación sociohistórica costarricense y las características de las acciones realizadas por el Estado en la atención de la pandemia por COVID-19. Además, las estrategias utilizadas por el gremio profesional ${ }^{1}$ de trabajo social, desde distintas instituciones públicas en un contexto de precarización de la política social y el mundo de trabajo, así como la agudización de las manifestaciones de la cuestión social.

Para la elaboración del presente artículo, se realizó una pesquisa bibliográfica y documental, utilizando fuentes primarias de información como noticias de periódicos, información del Colegio Profesional de Trabajo Social de Costa Rica y documentos públicos institucionales con datos sobre el tema.

El texto se encuentra compuesto por una introducción sobre las implicaciones de la COVID-19 en las relaciones sociales en el marco de una sociedad capitalista, particularmente en el contexto costarricense. Posteriormente, se realiza una aproximación a las respuestas por parte del Estado en la atención de la pandemia, finalizando con las intervenciones específicas que se han desarrollado en los distintos espacios socioocupacionales desde el trabajo social en el país.

Es de relevancia señalar que, desde el punto de partida asumido, se comprende la crisis sanitaria originada por la pandemia COVID-19 como una expresión de la crisis sistémica del capitalismo. Por esta razón, usamos la referencia a "crisis múltiples y multidimensionales", que en el fondo se constituyen en expresiones de la crisis de un sistema político, económico y social que es incompatible con la preservación de la vida en el planeta y de la propia especie humana. En este sentido, la pandemia por COVID-19 es una expresión de la crisis socioambiental y ecológica producto del Antropoceno y la relación de la sociedad con la naturaleza.

En este escenario, la gestión de las políticas públicas cobra una importancia fundamental para atender las expresiones más concretas de la crisis, así como visualizar alternativas ante las diversas formas de desigualdad que persisten en esta forma de organización social. Particularmente, en lo que concierne a los procesos en los que interviene trabajo social en este escenario en Costa Rica.

Es conveniente decir que el presente artículo consiste en una primera aproximación a la comprensión del rol que la profesión ha asumido en la atención de la pandemia desde las diversas instituciones del Estado, para la ejecución de las políticas sociales, emergentes, así como la adecuación de los procesos que ya existían de previo.

\footnotetext{
${ }^{1}$ El gremio profesional de Trabajo Social en Costa Rica, de acuerdo con datos del Colegio de Trabajadores Sociales 2021, está conformado por 6408 personas. Todas las personas profesionales para poder ejercer deben de estar agremiadas en el Colegio de Trabajadores Sociales de Costa Rica, el cual se divide en 8 sedes a nivel nacional.
} 
De esta manera, se pretende con este texto aportar a la reflexión y el análisis, sin agotar la discusión en torno al rol que la profesión tiene en un contexto en el que el papel del Estado y, por lo tanto, las políticas públicas deben ser analizadas y discutidas con miras a un proyecto ético-político transformador.

\section{Las implicaciones a nivel mundial y social de la COVID-19}

La pandemia originada por la COVID-19 encontró una sociedad muy golpeada debido a las consecuencias generadas por la propia reproducción del capital. Estas condiciones se venían expresando y agravando para grandes segmentos de la población, con expresiones crecientes de desigualdad y precarización. Solamente es necesario observar los datos de organismos internacionales, como el Programa de las Naciones Unidas para el Desarrollo (PNUD), para evidenciar esta situación.

Según datos del Informe del PNUD 2014,

más de 2.2 millones de personas continúan viviendo en situación de pobreza multidimensional... Esto significa que más del 15 por ciento de la población mundial permanece vulnerable a la pobreza multidimensional. Por otro lado, casi 80 por ciento de la población global no dispone de protección social ampliada. Cerca de 12 por ciento (842 millones) padecen de hambre crónica, y casi la mitad de los trabajadores -más de 1.5 millones- trabajan en régimen de empleo precario o informal. (PNUD, 2014, p. 15).

En el mismo informe del siguiente año, se confirma esta situación:

En 2015, 204 millones de personas en todo el mundo -incluyendo 74 millones de jóvenes (con edades entre 15 y 24 años)- estaban desempleadas. Cerca de 830 millones de trabajadores en regiones de países en desarrollo viven con menos de 2 dólares por día. En los países desarrollo, la mitad de los Trabajadores y sus respectivas familias viven con menos de 4 dólares por día. (PNUD, 2015, p.82).

Las desigualdades en el acceso al trabajo se expresan, además, en términos de género. Las mujeres no solo han tenido una menor participación en el mundo laboral (trabajo remunerado), sino que, cuando logran insertarse, lo hacen con condiciones de desigualdad persistentes. Según el informe de Naciones Unidas sobre los Objetivos de Desarrollo Sostenible 2019, en términos generales en el mundo el salario medio por hora de los hombres es un $12 \%$ más alto que el de las mujeres.

El promedio de la desigualdad salarial entre hombres y mujeres supera el 20 $\%$ en las ocupaciones directivas y profesionales, así como entre los artesanos y trabajadores de venta de artesanías, y los operadores y ensambladores en plantas de maquinaria. (ONU, 2019, p. 39).

Esta situación persiste y se agrava actualmente debido a la pandemia, que afecta de manera diferenciada las diversas regiones del mundo. Latinoamérica ha sido la región más golpeada en el empleo a causa de la crisis generada por la COVID-19, con una desocupación promedio de $11.1 \%$ y 28 millones de personas que, aunque trabajan, no ganan lo suficiente para mantener a sus familias por encima de la línea de pobreza, según la Organización Internacional del Trabajo (OIT, 2020). 
Este dato fue presentado el 11 de junio de este año, en el marco de la 109 Conferencia Internacional del Trabajo, realizada de manera virtual y en la que la OIT expuso su informe global.

Dicho informe evidencia que las personas de la región de América Latina y el Caribe experimentaron una caída más fuerte en la cantidad de horas trabajadas durante 2020. Asimismo, que se habría perdido el equivalente a más de 30 millones de empleos, tanto por las salidas de la fuerza de trabajo y la desocupación o inactividad, como por la reducción de horas trabajadas. Según las previsiones de la OIT, la tasa de desocupación regional fue de 8.0 \% en 2019, subió a 10.3 \% en 2020, y llegaría a 11.1 \% este 2021, lo que implicaría que la región tendrá 34 millones de personas buscando un puesto de trabajo sin conseguirlo (OIT, 2020).

El mismo informe menciona que para el 2022 se produciría una baja en la tasa a $8.9 \%$, pero estas cifras están expuestas a grandes niveles de incertidumbre y son dependientes de muchos factores. Adicionalmente, el informe mostró que la pobreza laboral aumentó en 5 millones de personas en el 2020, pasando de 23 a 28 millones de personas, de los cuales casi 9 millones están en condiciones de pobreza extrema.

Además, aunado al desempleo, se evidencia un aumento en el subempleo y la precarización del empleo

En el mundo casi 500 millones de personas trabajan menos horas remuneradas de las que desearían o no tienen suficiente acceso al trabajo asalariado, y para millones de personas es cada vez más difícil edificar mejores vidas basadas en sus posibilidades de trabajo. Existe un desajuste entre la oferta y la demanda de mano de obra, que se extiende más allá del desempleo -el cual ya suma unos 188 millones de personas-, pues hay una fuerte subutilización de trabajadores. Se calcula que unos 165 millones de personas no tienen suficiente trabajo remunerado. (OIT, 2020, p. 18).

Actualmente, la pobreza de las personas trabajadoras, definida como \$3.20 al día en términos de paridad del poder adquisitivo, afecta a una de cada cinco personas de la población activa mundial.

Tomando en cuenta que el agravamiento de las condiciones de reproducción de la clase trabajadora ya se evidenciaba de forma sistemática, donde la recesión económica mundial se manifiesta de forma particular en los países de América Latina y el Caribe, la pandemia por su parte, como se muestra en los datos citados antes, ha generado un aumento significativo de los índices de informalidad, precariedad y desempleo de forma exponencial. Esto debido a la proliferación de empleos intermitentes, ocasionales y flexibles, lo cual amplifica los ya alarmantes niveles de pobreza y desigualdad.

Según el informe Panorama Laboral 2020 de la OIT, el crecimiento económico estima una caída de $4.4 \%$ del nivel de actividad global y, en el caso de América Latina y el Caribe, la contracción económica es de aproximadamente un 8 \% del PIB para el año 2020, la mayor en el mundo. Respecto a la pérdida de horas de trabajo producto de la pandemia, la región es la más afectada. Se perdieron el 10.3 \% de los ingresos provenientes del trabajo de las personas de la región.

Otro hecho que resalta en el análisis de los impactos de la pandemia a la situación del trabajo es que la pérdida de horas de trabajo se relaciona especialmente con la no participación en la fuerza de trabajo, lo que significa personas que dejan de estar disponibles para trabajar. 
Por lo tanto, impacta en una reducción de la población económicamente activa, porque no hay búsqueda activa de trabajo.

A diferencia de crisis anteriores, en la inmensa mayoría de los casos la disminución de la ocupación a escala mundial en 2020 propició más salidas de la fuerza de trabajo que desocupación, e hizo que 81 millones de personas más salieran de la fuerza de trabajo y que 33 millones quedaran desocupadas. En consecuencia, la tasa de participación en la fuerza de trabajo a escala mundial se ha visto reducida en 2.2 puntos. (OIT, 2020, p. 10).

Las mujeres han sido más proclives que los hombres a salir de la fuerza de trabajo debido, por un lado, a la necesidad de asumir otras labores de reproducción de las condiciones de vida familiares, como son el cuidado de otras personas, las tareas educativas entre otras. Por otro lado, por las mismas limitaciones desiguales que impone el mundo del trabajo a las mujeres, lo cual produce un aumento en la desmotivación para la búsqueda activa de trabajo.

Según datos de la Comisión Económica para América Latina (Cepal) un 56.9 \% de las mujeres en América Latina y un 54.3 \% en el Caribe se encuentran ocupadas en sectores en los que se prevé un mayor efecto negativo en términos del empleo y los ingresos (Cepal, 2021, p.2). Entre estos sectores destacan el comercio, las industrias manufactureras, el turismo y el servicio doméstico; ocupaciones altamente feminizadas.

Algunos de los sectores que se encuentran en mayor riesgo no solo emplean a la mayoría de las mujeres ocupadas en la región, sino que además se caracterizan por altas tasas de informalidad, bajas remuneraciones y bajos niveles de calificación. (Cepal, 2021, p.4).

La preocupación que se refleja en estos datos es que necesariamente las políticas sociales que se implementan para atender la crisis, tanto en el corto como en el mediano y largo plazo, deben tener como eje transversal un enfoque de género, ya que las estimaciones actuales de la Cepal señalan una afectación de los avances de los últimos 10 años en la independencia económica de las mujeres. Lo anterior deteriora aún más las condiciones de las mujeres y constituye un freno para lograr que la sociedad transite hacia un mayor acceso de equidad social, fundamental dentro del proyecto ético-político con que debe comprometerse la profesión de trabajo social, según un posicionamiento teórico-metodológico que busque una transformación hacia una sociedad verdaderamente justa e igualitaria.

A pesar de que la situación generada por la pandemia ha afectado casi a todos los sectores de la población, la intensidad de la repercusión varía en gran manera entre las clases sociales. La pandemia golpea de forma más fuerte a las personas en condición de pobreza y exacerba la ya agudizada desigualdad.

Por ejemplo, las personas trabajadoras del sector informal se ven gravemente afectadas por las medidas de confinamiento, a diferencia de las personas que tienen condiciones laborales más estables. Las personas poco cualificadas no pueden respetar el llamado de "quédate en casa", ya que esto significa no solamente dejar de percibir su inestable ingreso, sino también otras manifestaciones ocasionadas por su condición estructural, como situaciones de hacinamiento, la falta de servicios básicos y demás situaciones generadas por su entorno.

En lo que se refiere a la política de salud, se ha generado un recrudecimiento de las condiciones de salud, ya que las personas que tenían alguna condición médica no reciben la atención adecuada por la emergencia. Esto debido a que la minada seguridad social no resiste la gran demanda presentada. Aunado a lo anterior, el acceso a la educación se ve restringido por 
la falta de conectividad y medios necesarios, según datos de la Unicef, “al 23 de marzo de 2020, alrededor de 154 millones de niños, niñas y adolescentes (más del $95 \%$ de los matriculados en la región) se encontraban temporalmente fuera de las escuelas, cerradas a causa de la COVID-19". (Unicef, 2020, párr. 1).

Además, las situaciones de violencia doméstica y maltrato infantil -las que se tiene conocimiento- han venido en aumento, a pesar de que los sistemas sociales de acompañamiento en estos casos han sido debilitados por las mismas medidas de distanciamiento y atención de la situación sanitaria. Respecto a este último aspecto, Castro y Rivera (2020) señalan

la labor desarrollada por docentes, al igual que por psicólogas, orientadoras y trabajadoras sociales que laboran en los centros educativos de las comunidades donde mayores situaciones de violencia contra niñas, niños y adolescentes se presentan, cumplen un papel esencial en la "primera línea" de detección de manifestaciones de violencia física, psicológica o sexual. En este momento, la imposibilidad de que esta población asista a sus instituciones implica que los procesos de acompañamiento se encuentren en la mayoría de los casos en suspenso y la ausencia que el recurso que estos profesionales representan para muchas niñas y niños, en edad escolar especialmente, les pone en una situación de mayor vulnerabilidad. (p. 96).

Este escenario sociopolítico y económico muestra a su vez, según los datos actuales de la gestión de la pandemia, una dificultad para lograr políticas públicas efectivas para la superación de la coyuntura actual. Según datos de la Organización Panamericana de la Salud, la región de América Latina y Caribe posee el $8.4 \%$ de la población mundial, registra el $20 \%$ de los casos y el $32 \%$ de las defunciones por COVID-19 en el planeta, para el 15 de junio 2021. Esto refleja la vulnerabilidad de los sistemas de salud y seguridad social de la región, así como el limitado acceso a medidas sanitarias que reduzcan la letalidad del virus, como es la vacunación, que para esta fecha se había inmunizado con esquema completo solamente a un $10 \%$ de la población (OPS, 2021), mientras que países como Estados Unidos y Reino Unido tienen un 45 \% de la población inmunizada. (OUR WORLD IN DATA, 2021).

Particularmente en Costa Rica, datos a la misma fecha muestran que la cantidad de personas contagiadas supera los 351000 ( $6.9 \%$ de la población total del país), de las que 4473 son defunciones, representando una tasa de letalidad de un $1.3 \%$. (MINSA, 2021). A su vez, se cuenta con un porcentaje de inmunización de un $15 \%$, a través de la vacunación. (OUR WORLD IN DATA, 2021).

En el siguiente apartado se incorpora el análisis sobre las implicaciones socioeconómicas que ha tenido esta situación en el país, así como las principales medidas de políticas sociales que se han desarrollado.

\section{La realidad costarricense: un panorama de Costa Rica en el contexto actual}

Antes de marzo de 2020, situaciones estructurales ya venían afectando a Costa Rica en términos macroeconómicos, como la situación fiscal del país y el aumento del desempleo, entre otros aspectos que han complicado la situación de las familias que habitan el territorio nacional. Estas situaciones se han agudizado producto de las medidas sanitarias, necesarias para desacelerar el avance de los contagios por COVID-19 en el país, y sus implicaciones económicas.

Al igual que en otros países de la región, en Costa Rica como una de las respuestas a la crisis estructural del capital, se ha evidenciado un desmonte en las garantías sociales y en los derechos 
sociales de la población. La contrarreforma del Estado se ha distinguido por la desmaterialización de la política social, direccionándola hacia la focalización y selectividad como forma generalizada a la atención de las manifestaciones de la cuestión social.

Aunado a esta tendencia, como medida para atender el déficit creciente en las finanzas públicas, la gestión estatal se ha orientado a la negociación de préstamos a organismos internacionales que inciden negativamente en las condiciones de vida de la clase trabajadora. Por ejemplo, una de las estrategias que ha implementado el Gobierno costarricense actual ha sido la adquisición de diferentes empréstitos, especialmente con el Fondo Monetario Internacional, tal y como se aprobó recientemente en el país ${ }^{2}$. Esto condiciona las acciones del Estado y el fondo público a largo plazo. Además, refuerza e intensifica la condición de dependencia en la que se encuentra el país, como parte de una región periférica del capitalismo mundial, así como direcciona enfáticamente para socavar la materialidad y las condiciones de vida de las personas trabajadoras.

A pesar de que existen recomendaciones de diversas organizaciones, como el ICEFI ${ }^{3}$ y OXFAM Internacional ${ }^{4}$, para atender la crisis fiscal en los países de la región centroamericana y generar oportunidades para el financiamiento de políticas sociales que atenúen los impactos de la crisis que atraviesa la región, las reformas fiscales que se ejecutaron en Costa Rica tanto previo a la crisis como durante su contención han tenido un carácter regresivo; por ejemplo, se ha creado un impuesto temporal al empleo, no así al capital ni las grandes fortunas.

Como se ha mencionado previamente, en Costa Rica el desempleo ya venía en aumento previamente a la pandemia, pasando de representar un $9.1 \%$ para 2017, a un $11.8 \%$ en 2019. En el contexto de la pandemia, esta situación se ha agravado, hasta alcanzar índices de desempleo entre los más altos de la región. Para el cuarto trimestre móvil 2020, según el Instituto Costarricense de Estadística y Censos (INEC) la tasa de desempleo fue de $20 \%$, lo que significa un aumento interanual de 7.6 p.p., comparado con el mismo periodo de 2019. Respecto a los sectores productivos más afectados y los empleos asociados a estos, se indica lo siguiente:

en los trabajadores asalariados disminuyó la población ocupada que gana menos de un salario mínimo y las personas con un rango de ingreso entre uno a menos de dos salarios mínimos (la diferencia de un año a otro fue de $159 \mathrm{mil}$ personas asalariadas), aunque el impacto no afecta los ingresos promedio del 2020, fueron los empleos de menor ingreso los que se perdieron de un año a otro, especialmente los empleos en ocupaciones elementales y de

\footnotetext{
2 “Desde su sede en Washington, el Directorio Ejecutivo del Fondo Monetario Internacional (FMI) anunció este lunes la aprobación de un acuerdo ampliado de 36 meses en el marco del Servicio Ampliado (SAF) para Costa Rica, por un monto equivalente a \$1.237,49 millones en Derechos Especiales de Giro (DEG) -o al $335 \%$ de la cuota correspondiente al paísunos US $\$ 1.778$ millones.

Esta aprobación “permite un desembolso inmediato equivalente a US \$296,5 millones, aproximadamente y complementa el apoyo de emergencia del FMI dado a Costa Rica en abril de 2020 por el $100 \%$ de la cuota, equivalente a US $\$ 521,7$ millones. Se espera que el acuerdo catalice otras ayudas financieras bilaterales y multilaterales", dijo la entidad en un comunicado". (SEMANARIO UNIVERSIDAD, 01 de marzo, 2021).

${ }^{3}$ Instituto Centroamericano de Estudios Fiscales (ICEFI). Agenda Fiscal Centroamericana 2021-2030. La realidad de Centroamérica: un diagnóstico comprehensivo.2021. Disponible en: https://icefi.org/sites/default/files/afca_icefi_2021_-_ volumen_i.pdf

${ }^{4}$ Cañete, Rosa. Democracias capturadas: El Gobierno de unos pocos. Mecanismos de captura de la política fiscal por parte de las élites y su impacto en la desigualdad en América Latina y el Caribe (1990-2017). Oxfam Internacional. 2018. Disponible en: https://oxfamilibrary.openrepository.com/bitstream/handle/10546/620600/rr-captured-democracies-161118-es.pd f;jsessionid=5493C3D645A9E6D8FCA743843574D5A0? sequence $=1$
} 
comercio y servicios principalmente. (INEC, 2021, p. 34, negrita incorporada por las autoras).

Es de preocupación el hecho de que el perfil de las personas en las que ha aumentado el desempleo son personas que, generalmente, pertenecen a los quintiles I y II, por lo que la reducción de ingresos en estas familias tiene un impacto directo en la incapacidad de atender sus necesidades básicas. En cuanto a los cambios en la ocupación, a partir del INEC (2021), se destaca el perfil de las personas que perdieron su empleo de un año a otro. En su mayoría son personas entre 15 a 24 años (28.4\%) y 25 a 34 años (26.1\%), con nivel educativo de primaria completa o menos (42.5\%), poseían un empleo permanente $(96.9 \%)$ y, mayoritariamente, se encontraban vinculadas al sector informal (58.1\%).

El resultado de la reducción de los ingresos en los hogares ha sido un crecimiento de la pobreza, llegando en el 2020 al índice de pobreza más alto de los últimos 10 años, según la Encuesta Nacional de Hogares (ENAHO) 2020, alcanzando un $26.2 \%$ de los hogares. Cabe ampliar respecto a este elemento, ya que los datos se refieren al porcentaje de hogares en condición de pobreza; sin embargo, dado que los hogares de más bajos ingresos generalmente por su composición son más numerosos,

La incidencia de la pobreza en términos de personas es de $30,0 \%$, en términos absolutos representa 1529255 personas en el 2020, esto equivale a un aumento de 6,1 pp. Respecto al 2019, es decir, 321874 personas más en condición de pobreza, lo cual se considera un cambio estadísticamente significativo. (INEC, 2020, p. 48).

Además, la ausencia de fuentes de ingreso familiar por medio del acceso al trabajo aumenta la dependencia de transferencias monetarias de parte del Estado, que, según datos de la misma fuente, pasó de representar un 1.8 \% de los ingresos por hogar para el año 2019, a tener un peso de $5.2 \%$ para el 2020 (INEC, 2020).

Esto significa, por un lado, que se ha generado en un momento de la pandemia una respuesta efectiva para atender necesidades de los hogares a través de transferencias económicas, sin embargo, los ingresos familiares han disminuido en todos los segmentos de población y en los hogares más dependientes de las políticas sociales significa mayores dificultades.

Sobre estas medidas se abordará en el siguiente apartado.

\section{Medidas de política para atender la situación producto de la crisis sanitaria en Costa Rica}

Con respecto a la pandemia, en Costa Rica, el 06 de marzo del 2020 se confirma el primer caso de coronavirus en el país. Cinco días después, la Junta Directiva de la Caja Costarricense del Seguro Social (CCSS) aprobó una nueva modalidad de incapacidad para personas con síntomas de COVID-19, por medio de una orden sanitaria para el aislamiento domiciliario.

En el mismo mes, el 16 de marzo, el Gobierno costarricense se manifiesta y anuncia una serie de medidas con el fin de mitigar el impacto a nivel económico y social que previeron con las implicaciones generadas por la pandemia. ${ }^{5}$

${ }^{5}$ El Gobierno declara estado de emergencia nacional, impide llegada de personas extranjeras y se suspende lecciones en todos los centros educativos del país (Comunicado de prensa, Presidencia de la República, 16 de marzo, 2020). 
Como parte de estas medidas, se emite el Decreto Ejecutivo N²4227-MP-S, en el cual se declara "Estado de Emergencia Nacional". A partir de este decreto, se suspenden de forma temporal las actividades de naturaleza judicial, las lecciones en centros educativos públicos y privados y se cierran las fronteras marítimas, aéreas y terrestres.

Además, se aprueba el proyecto de ley N²1.854 denominado "Ley de Autorización de Reducción de Jornadas de Trabajo ante la Declaratoria de Emergencia Nacional" y se anuncian las medidas tomadas por la Mesa de Protección Social ante la emergencia generada por la pandemia.

Entre las medidas mencionadas, se incluye la protección social de distintos grupos poblacionales históricamente vulnerables como la niñez, mujeres, personas con discapacidad, personas adultas mayores, pueblos originarios, personas en situación de calle y familias en condición de pobreza.

Mediante la Ley de Protección a las Personas Trabajadoras durante la Emergencia por la Enfermedad COVID-19, Ley Nº 9840 del 22 de abril de 2020, se creó un subsidio para la atención de la condición de desempleo, suspensión temporal del contrato de trabajo o reducción de jornadas laborales, en favor de las personas trabajadoras del sector privado, los trabajadores temporales o informales y los trabajadores independientes que vieran sus ingresos afectados a consecuencia de la entrada en vigencia del Decreto Ejecutivo N 42227-MP-S del 16 de marzo de 2020 y de las medidas adoptadas por las autoridades nacionales durante la fase de respuesta y rehabilitación de la emergencia por el virus.

Esta política se definió según el considerando IV del Decreto Ejecutivo № 42329-MTSS-MDHIS como "una transferencia monetaria extraordinaria y temporal para contribuir con la protección social de las personas afectadas por el cambio en sus condiciones laborales y/o de ingresos como consecuencia de la Emergencia Nacional provocada por el COVID-19". (Costa Rica, 2021).

Este se estableció como un subsidio temporal y estuvo vigente entre los meses de abril 2020 y enero 2021. Según datos de marzo 2021 publicados por el Ministerio de Trabajo y Seguridad Social (MTSS), 724330 personas habían recibido al menos un desembolso del programa, de los cuales un $38.24 \%$ corresponde a personas catalogadas como "trabajador independiente", un $23.19 \%$ a personas que fueron despedidas de sus empleos y un $22.9 \%$ a personas trabajadoras temporales o informales (MTSS, 2021). El resto son personas que han visto sus jornadas reducidas o reportan suspensión temporal de su contrato de trabajo.

Clasificando a las personas por su nivel educativo, "el 72.1\% de las solicitudes registradas en la plataforma Proteger corresponde a personas con secundaria incompleta o menos, el $13.8 \%$ corresponde a personas que completaron la secundaria y el restante $14.1 \%$ de aquellas personas que cuentan con niveles de educación vocacional, técnica o universitaria (tanto completa como incompleta)". (COSTA RICA, 2021, p. 12).

Lamentablemente, esta acción ha sido muy limitada para atender los efectos que la pandemia ha tenido en las personas trabajadoras y sus familias. A su vez, en el país han sido sumamente restringidas las acciones para acompañar a las empresas, especialmente las medianas y pequeñas empresas de capital nacional. Esto genera la necesidad de discutir medidas que aporten a generar transferencias que efectivamente den soporte a estas personas, ya que la crisis económica y social que atraviesa el país no ha terminado. Asimismo, acciones que permitan, particularmente, la recuperación del empleo.

Todo este marco contextual influye en el trabajo profesional de las personas trabajadoras sociales, por lo que es necesario evidenciar las acciones de este gremio en la coyuntura generada por la pandemia. 


\section{Trabajo social en la primera línea de intervención ante la pandemia}

En atención a la crisis sanitaria por la COVID-19, surge la necesidad de localizar tanto a las instituciones y organizaciones como a las personas profesionales en trabajo social que han atendido de diversas formas la coyuntura actual del país y el mundo. Dado que esta pandemia por ser una situación atípica y nunca abordada por la profesión provoca retos importantes, se intenta generar un insumo que sintetice las acciones realizadas.

\section{Caracterización general de las personas profesionales en trabajo social en Costa Rica}

El trabajo social, como profesión vinculada orgánicamente a la complejidad de la sociedad capitalista desde su conformación histórica hasta la actualidad, se va reconfigurando a partir de las necesidades expresadas por el actual modo de producción.

Es con el auge de la política de corte reformista costarricense en la década de 1940, que se propician las condiciones para la constitución de la profesión en este país. Se demanda por las propias instituciones y, posteriormente, vinculada a la instancia de formación profesional.

La tendencia de la profesión desde su nacimiento, la posiciona como eminentemente técnica, como lo expresa el siguiente autor.

LagénesisdelaEscuelamantuvoensusprimerosañosun perfilhegemónicamente técnico, marcado por su origen, pues las demandas del mundo del trabajo fueron la preocupación fundamental bajo la cual surgió, marcada por una falta de legitimidad académica, es decir no fue una preocupación de la Universidad sino de la institucionalidad. (VILLALOBOS, 2014, p.6).

En las décadas siguientes, el país vivió cambios tanto en los procesos productivos como en la forma de intervención del Estado en las manifestaciones de la cuestión social, lo que influenció el desarrollo del trabajo social en Costa Rica.

Como lo menciona Sánchez y Torres (2020, p.12),

Desta maneira, podem-se observar as tendências teóricas que têm direcionado a formação do Trabalho Social na Costa Rica, ainda presentes na contemporaneidade, porquanto são hegemônicas as posturas de base positivista. Embora o curso de Trabalho Social da Universidade de Costa Rica, em alguns contextos, haja desenvolvido projetos de intenção de ruptura com importantes avanços, isso não se tem consolidado de forma ampla, generalizada e sistemática. Persiste uma forte herança de posturas conservadoras.

Se evidencia así que el proceso de formación y actuación del trabajo social durante la penúltima década del siglo XX sufrió una serie de cambios cada vez más articulados a la lógica neoliberal y la contrarreforma. Estas últimas trajeron consigo una fuerte tendencia a la mercantilización y privatización tanto de los servicios sociales como de la formación profesional.

Actualmente, el gremio profesional se encuentra compuesto por aproximadamente 6408 profesionales, constituido mayoritariamente por mujeres (91.3\%) y con un mayor porcentaje de personas entre los 36 y 64 años (45 \%). (COLTRAS, 2021, p. 6).

En este contexto de precarización laboral y desmejoramiento de las condiciones de vida de la población, trabajo social no se ve exento, ya que es una profesión que tiene un alto índice 
de desempleo, con cifras de más del $18 \%$ de las personas profesionales en esta condición. (CONARE, 2018).

Las personas profesionales que se encuentran laborando se ubican mayoritariamente en instituciones públicas (77\%), seguido por la contratación de profesionales en organizaciones no gubernamentales (13\%) y empresas privadas (7 \%). (COLTRAS, 2020).

Dentro de los niveles de intervención y atención, las personas profesionales en trabajo social se encuentran tanto a nivel de instituciones centrales, que ejecutan políticas sociales de salud, educación, justicia, infancia, entre otras, como en oficinas locales en el nivel municipal donde se ejecutan políticas dirigidas a poblaciones específicas: mujeres, personas adultas mayores, niñez y adolescencia, etcétera.

Tomando en cuenta estos datos, tener un panorama general de las acciones y estrategias que se han desarrollado desde las distintas instituciones y organizaciones donde laboran las personas profesionales en trabajo social es difícil, especialmente por las múltiples actividades realizadas desde nuestra diversa profesión. Aunado a la sobrecarga de las labores realizadas, lo cual limita las posibilidades de sistematizar, como parte de los procesos de trabajo, la práctica profesional en la mayoría de los espacios sociolaborales.

A pesar de lo anterior, a partir de la información suministrada por el Colegio Profesional de Trabajo Social de Costa Rica (Unidad de Investigación, 2021), se han podido registrar actividades importantes que la profesión viene asumiendo en el marco de la pandemia, las cuales podemos agrupar en los siguientes puntos:

\section{Divulgación de información y prevención}

Una de las tareas más activas ha sido divulgar información preventiva sobre la pandemia y temas relacionados. Se realizan múltiples acciones de elaboración de materiales con información sobre prevención en torno al tema de la COVID-19, situaciones familiares, información específica de la prestación de servicios de la institución, entre otros. Este material es divulgado por diferentes medios, como correo electrónico, redes sociales y diversas plataformas, con el fin de ser socializado a un amplio número de la población usuaria de los servicios.

\section{Continuidad en la atención de servicios sociales}

Con el fin de generar una continuidad en los servicios y garantizar los derechos de las personas usuarias, profesionales en trabajo social de distintas instituciones, especialmente las de primera línea, han ideado estrategias y han modificado las formas de intervención para ajustarse a las condiciones coyunturales.

Por ejemplo, en diferentes espacios laborales, como las áreas de salud, educativa, entre otras, se ha virtualizado la atención, es decir, se continúa la consulta por medios digitales, videollamadas o llamadas telefónicas. Lo anterior, permite brindar seguimiento a situaciones que generan vulnerabilidad y riesgo como violencia intrafamiliar, imposibilidad de satisfacer necesidades básicas que comprometan su salud física y mental, además del fortalecimiento de las redes de apoyo, entre otros, especialmente en personas diagnosticadas positivas COVID-19 y sus familias. 


\section{Elaboración de propuestas y protocolos}

Desde algunas instituciones se han elaborado protocolos para direccionar el trabajo profesional, como en el Ministerio de Educación Pública y la Coordinación Nacional de Trabajo Social de la CCSS, donde se han realizado lineamientos y protocolos de atención específicos para trabajo social durante la pandemia. Esto ayuda a las personas profesionales a orientar sus procesos de trabajo y se considera una buena práctica para implementar a nivel general.

Estos protocolos se han realizado con apoyo de instancias investigativas como las universidades, con el fin de buscar una orientación y regulación técnica de la intervención social que realizan los Servicios de Trabajo Social dirigida a las personas con COVID-19, sus familias y redes de apoyo.

Uno de los principales empleadores de las personas profesionales de este gremio es la Caja Costarricense del Seguro Social (CCSS), donde se encuentran trabajando más de 538 profesionales. Según información suministrada por la Dirección de Desarrollo de Servicios de Salud-Gerencia Médica, se indican las acciones ejecutadas por las personas profesionales en trabajo social del Régimen de Salud, que actualmente se encuentran atendiendo la emergencia por COVID-19 en la institución.

Las acciones son realizadas por las personas profesionales de trabajo social de los Servicios de Trabajo Social en los tres niveles de atención de la Red de Servicios. Actualmente, estas personas profesionales forman parte de 135 equipos multidisciplinarios que atienden en las 106 áreas de salud y hospitales existentes, brindando atención con una direccionalidad psicosocial a las personas con COVID-19 y sus familias.

Entre las acciones realizadas sobresalen la continuidad en la prestación de servicios a pacientes y sus familias o redes de apoyo, que demandan servicios para la atención de las implicaciones sociales asociadas a una situación de salud-enfermedad en los tres niveles de atención de la Red de Servicios.

A su vez, las personas con diagnóstico positivo por COVID-19 y sus familias son atendidas por el área de Trabajo Social de Hospitales y Áreas de Salud de la CCSS, mediante teleconsulta (llamada telefónica o video llamada). Esto ha permitido identificar, prevenir y dar seguimiento a situaciones de riesgo como violencia intrafamiliar, imposibilidad de satisfacer necesidades básicas que comprometan su salud física y mental, manejo del distanciamiento físico en el ámbito familiar, fortalecimiento de las redes de apoyo, entre otros.

En cuanto a manejo de poblaciones específicas, en el caso de población adulta mayor se ha priorizado su atención por parte de todos los servicios de trabajo social de la CCSS. Además, se realiza una intervención social con la persona cuidadora o localización de otros recursos de apoyo (hogares para adultos mayores, otros familiares o recursos comunales) que se requieran.

Con el fin de mantener el servicio y buscando la promoción de la salud integral, las personas profesionales en trabajo social de la CCSS reinventaron su intervención, atendiendo especialmente a las familias que, ante el deceso de la persona proveedora, perdieron el ingreso familiar, o bien, en las situaciones donde requieren de una ayuda estatal por su condición económica.

Por otro lado, en cuanto a las profesionales vinculadas al Ministerio de Educación Pública, las acciones realizadas son variadas. Desde el Departamento de Vida Estudiantil, se realizó material de apoyo y divulgación de información para los equipos interdisciplinarios. 
Además, desde la Unidad para la Permanencia, Reincorporación y Éxito Educativo, se elaboró un "Protocolo de reinserción 2020", el cual define los lineamientos para la reincorporación de personas al sistema educativo.

Entre estos lineamientos, se enmarcan las acciones que deben de estar centradas en una educación de calidad para todas las personas, particularmente, para las poblaciones que se encuentran en condiciones más vulnerables y de riesgo en nuestro país. Para esto, se menciona que el sistema educativo ofrece, en las distintas regiones del país, diversas ofertas educativas, para que la población pueda reincorporarse y concluir exitosamente sus estudios.

Se sugieren ciertas acciones a realizar como localizar a las personas, llamadas telefónicas al hogar, visitas domiciliares, activación de redes de estudiantes y familias que sirvan como enlace de localización, activación de redes interinstitucionales en caso de que no se logre localizar a la persona estudiante o personas encargadas legales mediante las acciones anteriores y realizar la coordinación con actores claves como Asistentes Técnicos de Atención Primaria (ATAPs), personas funcionarias del IMAS, cogestores sociales, Sistema de atención integral intersectorial del desarrollo del niño y la niña (SAIID) y otros para facilitar y actualizar el contacto de localización de la persona o la familia.

De esta forma, los lineamientos planteados constituyen estrategias para atender la reinserción, permanencia y vulnerabilidad de las personas estudiantes en los contextos educativos a distancia durante la pandemia. Sin embargo, la realidad objetiva y material de esta población ha evidenciado que algunas de estas estrategias propuestas no se puedan llevar a cabo, tomando en cuenta la pobreza y desigualdad que vive un gran porcentaje de estudiantado matriculado en las escuelas y colegios públicos del país.

Según datos del Ministerio de Educación Pública, desde marzo del año pasado, donde se ordenó el cierre de las clases en modalidad presencial, hasta diciembre de este mismo año, más de 10000 personas estudiantes habían sido excluidas del sistema educativo. Reto de particular importancia para las profesionales en trabajo social insertas en los equipos interdisciplinarios del MEP, que se ubican en comunidades excluidas del país.

Estas referencias son algunas de las evidencias de los procesos de apoyo que trabajo social ha realizado en el contexto de pandemia por COVID-19 en Costa Rica, buscando hacer reflexión y sistematizar la práctica profesional en un país donde la profesión históricamente ha estado asociada al Estado y a su rol de intervención, particularmente en un contexto de crisis como el actual.

Es de fundamental importancia que estas reflexiones puedan seguirse alimentando mediante la discusión gremial e interdisciplinar.

\section{Consideraciones finales}

En un contexto de resistencia al retroceso civilizatorio en el que vivimos, donde se criminaliza cada vez más la pobreza y existe una regresión de los derechos sociales y los ataques a la seguridad social cada vez más violentos, se hace necesario evidenciar las estrategias y logros de la categoría profesional.

Como profesionales en trabajo social es necesario evidenciar nuestras intervenciones en esta coyuntura, reflexionar y debatir no solamente para conocer las prácticas realizadas desde otras instituciones, sino para posicionarnos y proponer estrategias como colectivo profesional. 
La reestructuración del Estado y el desmonte de la política social ha vulnerabilizado nuestras condiciones laborales y cada vez existen más mecanismos legales que atentan contra las personas profesionales de nuestro gremio y a la clase trabajadora en general.

En los últimos tiempos hemos visto como la disminución de los derechos se ha materializado, reflejándose en el congelamiento del pago de los complementos laborales, por ejemplo, y el estancamiento salarial en las personas funcionarias públicas y privadas. Así como la poca injerencia que tenemos como colectivo profesional en puestos de toma de decisiones y la precarización de las condiciones laborales, agudizadas en el contexto de la pandemia.

Trabajo social es una profesión que desde su conformación histórica se encuentra tensionada por las demandas tanto del capital como de las personas que son atendidas en los diferentes espacios laborales; situaciones que se presentan cada vez más agudizadas y ampliadas. Esto trae consigo una necesidad de la búsqueda constante de estrategias, a partir de la capacidad de las personas profesionales en trabajo social de aprehender las dimensiones colocadas en la realidad con las posibilidades materiales presentes en la institucionalidad donde se encuentra ubicada.

Específicamente, en el contexto generado por la pandemia, entre los principales desafíos operativos se encuentra la pérdida del contacto humano, sustituida por herramientas tecnológicas que traen consigo una serie de limitaciones en nuestro actuar profesional.

Adicionalmente, las medidas de contención de la crisis no han sido suficientes para atender la precarización de condiciones de vida de grandes segmentos de población, con especial énfasis en hogares que dependían de los ingresos generados por mujeres, donde las tareas de los cuidados se han recargado y dificultan la posibilidad de desarrollar labores de manera remunerada.

Es evidente que, para la erradicar la desigualdad y la pobreza, es necesario un cambio radical en la forma de producción y reproducción del modo de producción en el que vivimos. Sin embargo, nos parece importante generar una práctica reflexiva y transformadora, en el sentido de fortalecer las estrategias profesionales con un horizonte a favor de la mejoría substancial de las condiciones de vida de la clase que vive del trabajo, como fundamento del proyecto éticopolítico que direcciona nuestro accionar como profesionales.

\section{Referencias}

CASTRO, Natalia.; RIVERA, Laura. ¿Cómo vive la niñez y la adolescencia la pandemia generada por COVID-19 desde las desigualdades socioeconómicas? Boletín 9 Infancia, Instituto Interamericano del Niño y la Niña, OEA, p.91-100, junio 2020. Disponible en: https://issuu.com/ institutointeramericanodelninolanin/docs/boletin_9_espa_ol_final

COLEGIO DE TRABAJADORES SOCIALES DE COSTA RICA (Costa Rica). Informe de consulta de condiciones laborales de las personas profesionales en Trabajo Social- 2020. San José, 2020.

COLEGIO DE TRABAJADORES SOCIALES DE COSTA RICA (Costa Rica). Memoria de Junta Directiva 2019-2021. San José, 2021.

COMISIÓN ECONÓMICA PARA AMÉRICA LATINA Y EL CARIBE (Cepal). La Agenda 2030 para el Desarrollo Sostenible en el nuevo contexto mundial y regional: escenarios y proyecciones en la presente crisis. Santiago, 2020.

COMISIÓN ECONÓMICA PARA AMÉRICA LATINA Y EL CARIBE (Cepal). La autonomía económica de las mujeres en la recuperación sostenible y con igualdad. Informe especial COVID-19 \#9. 10 
de febrero de 2021. Disponible en: https://www.cepal.org/es/publicaciones/46633-la-autonomiaeconomica-mujeres-la-recuperacion-sostenible-igualdad

CONSEJO NACIONAL DE RECTORES. (CONARE) Seguimiento de la condición laboral de las personas graduadas de pregrado 2014- 2016 de las universidades costarricenses: incluye implicaciones laborales durante la pandemia. San José, 2018.

COSTA RICA. Decreto Ejecutivo № 42329-MTSS-MDHIS. Reforma Creación del Bono Proteger. 29 de abril, 2020. Disponible en: http://www.pgrweb.go.cr/scij/Busqueda/Normativa/Normas/nrm texto_completo.aspx?param1=NRTC\&nValor1=1\&nValor2=91152\&nValor3=120301\&strTipM=TC

COSTA RICA..Noveno Informe Mensual de Seguimiento a la Ejecución del Bono Proteger. 2021. Disponible en: http://www.mtss.go.cr/elministerio/despacho/covid-19-mtss/plan_proteger/archivos/ noveno_informe_proteger.pdf

COSTA RICA.. Gobierno declara estado de emergencia nacional, impide llegada de extranjeros y se suspende lecciones en todos los centros educativos del país. 2020. Disponível em: https://www. presidencia.go.cr/comunicados/2020/03/gobierno-declara-estado-de-emergencia-nacional-impidellegada-de-extranjeros-y-se-suspende-lecciones-en-todos-los-centros-educativos-del-pais/. Acesso em: 28 set. 2021.

INSTITUTO NACIONAL DE ESTADÍSTICA Y CENSOS (INEC). Encuesta Nacional de Hogares julio 2020: Resultados generales [recurso electrónico]. Año 9. San José, 2020. Disponible en: https://www.inec. $\mathrm{cr} /$ sites/default/files/documetos-biblioteca-virtual/renaho2020.pdf

INSTITUTO NACIONAL DE ESTADÍSTICA Y CENSOS (INEC).. Encuesta Continua de Empleo, IV Trimestre 2020. San José, 2021. Disponible en: https://www.inec.cr/sites/default/files/documetosbiblioteca-virtual/reeceivt2020.pdf

MARTÍNEZ FRANZONI, Juliana y SÁNCHEZ-ANCOCHEA, Diego. Pactos sociales al servicio del bienestar en América Latina y el Caribe: ¿qué son y qué papel tienen en tiempos de crisis?, Documentos de Proyectos (LC/TS.2020/169), Santiago, Comisión Económica para América Latina y el Caribe (CEPAL), 2020. Disponible en: https://www.cepal.org/sites/default/files/publication/ files/46527/S2000750_es.pdf

MINISTERIO DE SALUD (MINSA). Situación nacional Covid-19, 2021. Disponible en: https://www. ministeriodesalud.go.cr/index.php/centro-de-prensa/noticias/741-noticias-2020/1725-situacionnacional-covid-19

MINISTERIO DE TRABAJO Y SEGURIDAD SOCIAL (MTSS). Datos sobre el Bono Proteger. 2021. Disponible en: http://www.mtss.go.cr/elministerio/despacho/covid-19-mtss/plan_proteger/datos/ enero_21/115\%20Datos\%20Proteger\%205\%20marzo\%202021.jpg

ORGANIZACIÓN INTERNACIONAL DEL TRABAJO (OIT). Panorama Laboral 2020 América Latina y el Caribe. Lima: OIT / Oficina Regional para América Latina y el Caribe, 2020. 204 p.

ORGANIZACIÓN PANAMERICANA DE LA SALUD (OPS). Informes de situación de la COVID-19. 2021. Disponible en: https://www.paho.org/es/informes-situacion-covid-19

OUR WORLD IN DATA. Statistics and Research Coronavirus (COVID-19) Vaccinations. 2021. Disponible en: https://ourworldindata.org/covid-vaccinations

PNUD. United Nations Development Programme. Human Development Report 2014: Sustaining Human Progress - Reducing Vulnerabilities and Building Resilience. New York, 2014. Disponible en: http://hdr.undp.org/en/content/human-development-report-2014 
PNUD. United Nations Development Programme. Human Development Report 2015: Work for Human Development. New York, 2015. Disponible en: http://hdr.undp.org/en/content/humandevelopment-report-2015

SANCHEZ, Mariangel; TORRES, María Adriana da Silva. A FORMAÇÃO ACADÊMICO-PROFISSIONAL NO SERVIÇO SOCIAL/ TRABALHO SOCIAL: as especificidades do brasil e da costa rica. In: BORGES, Maria Virginia Amaral; HAURADOU, Gladson Rosas. Lutas, trabalho, “questão social” e Serviço Social. Maceió: Edufal, 2019. p. 1-163.

SEMANARIO UNIVERSIDAD (Costa Rica).FMI aprueba un acuerdo ampliado por US\$ 1.778 millones para Costa Rica. 2021. Disponível em: https://semanariouniversidad.com/pais/fmi-aprueba-unacuerdo-ampliado-por-us-1-778-millones-para-costa-rica/. Acesso em: 28 set. 2021.

UNICEF. COVID-19: Más del 95 por ciento de niños, niñas y adolescentes está fuera de las escuelas en América Latina y el Caribe. 23 Marzo 2020. Disponible en: https://www.unicef.org/ lac/comunicados-prensa/covid-19-mas-del-95-por-ciento-de-ninos-y-ni\%C3\%B1as-esta-fuera-delas-escuelas

VILLALOBOS, Manuel Antonio. RECONCEPTUALIZACIÓN A LA TICA: APUNTES HISTÓRICOS PARA EL TRABAJO SOCIAL COSTARRICENSE. Rev. Ciencias Sociales, San José, v. 1, n. 159, p. 57-71, 2018. 